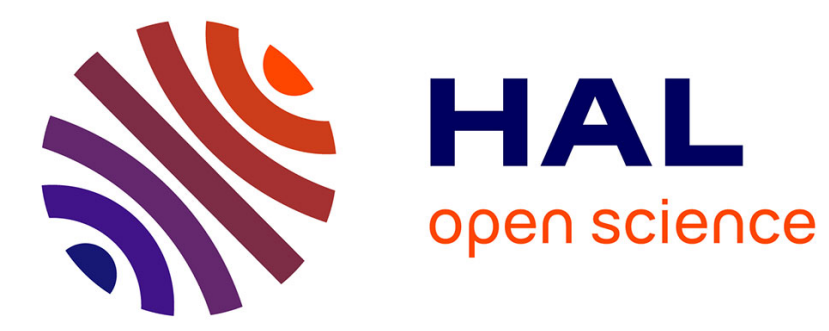

\title{
Modélisation et commande d'une hydrolienne équipée d'une génératrice asynchrone double alimentation
}

Seifeddine Benelghali, Mohamed Benbouzid, Jean Frédéric Charpentier

\section{To cite this version:}

Seifeddine Benelghali, Mohamed Benbouzid, Jean Frédéric Charpentier. Modélisation et commande d'une hydrolienne équipée d'une génératrice asynchrone double alimentation. European Journal of Electrical Engineering, 2010, 13 (2), pp.161-178. hal-00728342

\section{HAL Id: hal-00728342 \\ https://hal.science/hal-00728342}

Submitted on 5 Sep 2012

HAL is a multi-disciplinary open access archive for the deposit and dissemination of scientific research documents, whether they are published or not. The documents may come from teaching and research institutions in France or abroad, or from public or private research centers.
L'archive ouverte pluridisciplinaire HAL, est destinée au dépôt et à la diffusion de documents scientifiques de niveau recherche, publiés ou non, émanant des établissements d'enseignement et de recherche français ou étrangers, des laboratoires publics ou privés. 


\title{
Modélisation et commande d'une hydrolienne équipée d'une génératrice asynchrone double alimentation
}

\author{
Seif Eddine Ben Elghali* — Mohamed Benbouzid* Jean-Frederic \\ Charpentier**
}

* Université de Brest, EA 4325 LBMS

IUT de Brest-Rue de Kergoat, CS 93837, F-29238 Brest Cedex 03

\{Seif-Eddine.Benelghali,Mohamed.Benbouzid\}@univ-brest.fr

** Ecole Navale, EA 3634 IRENav

BCRM de Brest, Ecole navale et groupe des écoles du Poulmic

CC 600, Lanvéoc, F-29240 Brest Cedex 9

jean-frederic.charpentier@ecole-navale.fr

RÉSUMÉ. Cet article traite de la modélisation et de la commande d'un système de récupération de l'énergie des courants de marée; à savoir une hydrolienne équipée d'une génératrice asynchrone double alimentation. Dans un premier temps et dans le but de pouvoir évaluer les performances et la dynamique de l'hydrolienne, dans différentes conditions de fonctionnement, un modèle multiphysique est implanté sous Matlab/Simulink®. Ensuite, une stratégie de commande MPPT sans capteur de vitesse de la génératrice asynchrone est proposée. Cette stratégie est éprouvée en regard de l'effet de la houle qui est considéré comme le plus à même de perturber le modèle de la ressource. La modélisation multi-physique et la commande à vitesse variable ont été testés sur une hydrolienne équipée d'une génératrice asynchrone double alimentation de 7,5 $\mathrm{kW}$ et en utilisant des données réelles du site du Raz-de-Sein (Bretagne) pour l'année 2007.

ABSTRACT. This paper deals with the modeling and the sensorless control of a variable speed DFIG-based marine current turbine. The proposed MPPT sensorless control strategy relies on the resource and the marine turbine models that were validated by experimental data. The sensitivity of the proposed control strategy is analyzed regarding the swell effect as it is considered as the most disturbing one for the resource model. Tidal current data from the Raz-de-Sein (Brittany, France) are used to run simulations of a 7.5-kW prototype over various flow regimes. Simulation results are presented and fully analyzed.

MOTS-CLÉS: courants de marées, énergie hydrocinétique, hydrolienne, modélisation, commande, génératrice asynchrone double alimentation.

KEYWORDS: marine current turbine, tidal energy, modeling, control, doubly-fed induction generator (DFIG). 


\section{Introduction}

Les énergies marines peuvent contribuer à une part significative des nouvelles sources d'énergie n'émettant pas de gaz à effet de serre. Leur exploitation raisonnée permet de produire de l'électricité exportable à terre. Ces énergies renouvelables théoriquement exploitables sont nombreuses et variées. En effet, la mer est un fluide riche en flux énergétiques qui peuvent être exploités sous diverses formes. On peut citer par exemple, les énergies thermiques, hydrocinétiques (vagues et courants) et osmotiques.

Le développement de la plupart de ces énergies connaît plusieurs difficultés d'ordre économique et technologique qui sont dues essentiellement aux nombres réduits de zones économiquement exploitables et aux coûts élevés des techniques proposées (IEEE, 2005). Cependant, l'énergie hydrocinétique des courants de marée, que l'on peu récupérer grâce à des machines appelées hydroliennes, connaît un intérêt considérable par les chercheurs et les industriels vu la grande similarité des technologies utilisées avec les éoliennes (Johnstone et al., 2006). Les systèmes hydroliens se développent ainsi plus rapidement car on peut désormais s'appuyer sur des techniques fiables et éprouvées. De plus, le potentiel énergétique mondial de cette énergie est estimé à une puissance de $100 \mathrm{GW}$, ce qui représente un gisement énergétique non négligeable et très prometteur. Avec une ressource hydrocinétique avoisinant les $6 \mathrm{GW}$ répartie entre le Raz-Blanchard $(3 \mathrm{GW})$, Fromveur $(2 \mathrm{GW})$ et Raz-de-Sein $(1 \mathrm{GW})$, la France est l'un des pays les plus prometteurs en Europe occidentale après le Royaume Uni (10 GW) (figure 1).

Il est cependant à noter que dans ce domaine, aucune technologie de référence n'a encore émergé et que l'on constate à l'examen des projets actuels un foisonnement de technologies diverses (Ben Elghali et al., 2008 ; Benbouzid et al., 2007).

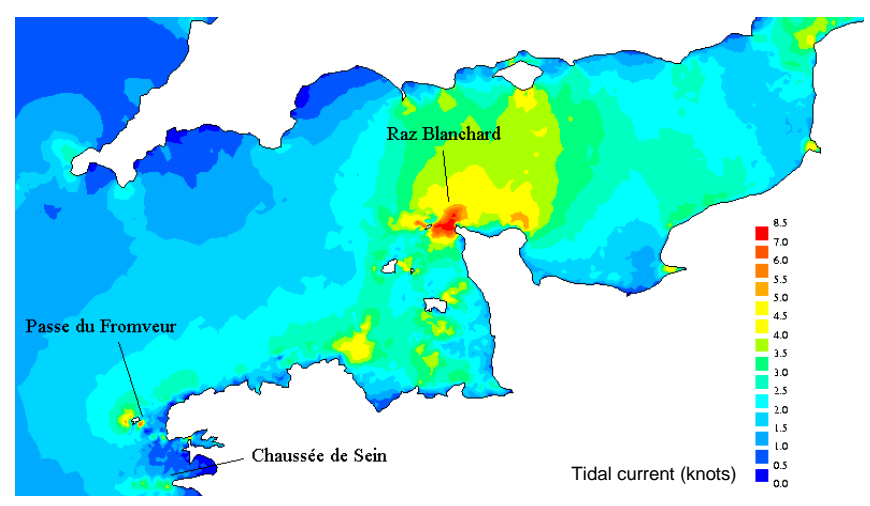

Figure 1. Carte des littoraux breton et normand 
Afin d'être capable d'une part de choisir une technologie adaptée à un site particulier et d'autre part de tester des lois de régulation d'un système hydrolien, il apparaît nécessaire de disposer d'un outil de simulation capable de modéliser l'ensemble de la chaîne de conversion d'énergie et de prévoir ces performances (Myers et al., 2005). Le travail présenté dans cet article se veut une présentation de ce type d'outil prédictif. Après avoir décrit les différents modèles employés, nous illustrons notre présentation par une validation concrète de notre outil de simulation où nous évaluerons l'énergie pouvant être extraite par une hydrolienne à hélice utilisant une génératrice asynchrone à double alimentation et placée dans le site du Raz-de-Sein.

\section{Modélisation de l'hydrolienne}

Le schéma global de la modélisation d'une chaîne de conversion d'énergie hydrocinétique connectée au réseau électrique est décrit par la figure 2.

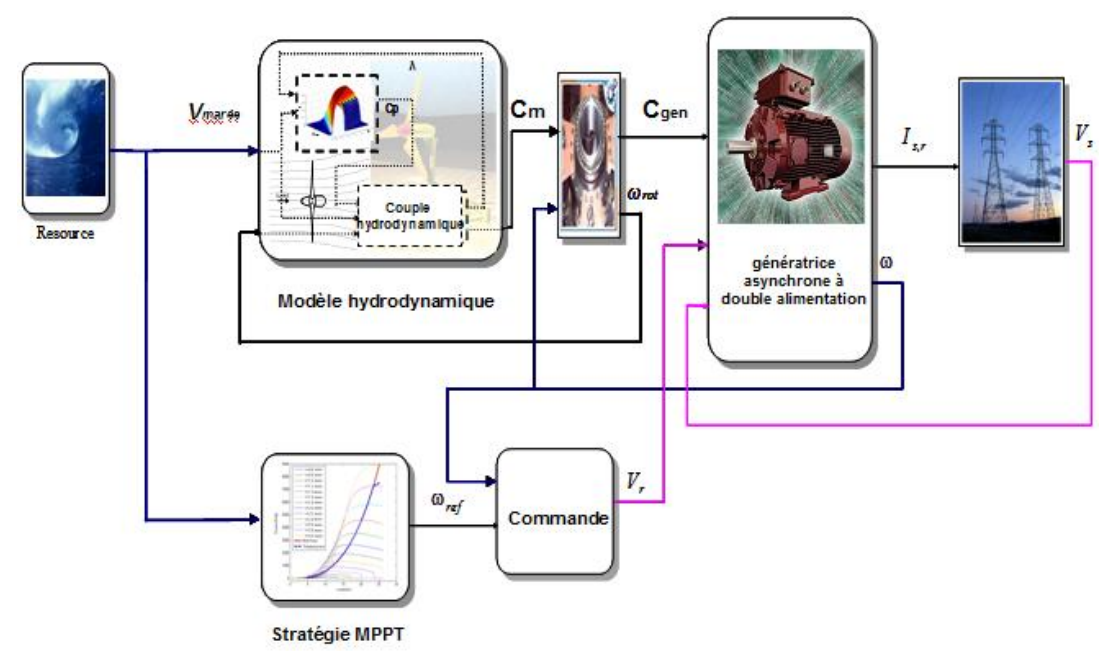

Figure 2. Schéma global d'une chaîne de conversion hydrocinétique utilisant une hydrolienne

\subsection{Modélisation de la ressource}

\subsubsection{Potentiel de la ressource}

La puissance cinétique totale disponible sur la turbine d'une l'hydrolienne est donnée par (Couch et al., 2006 ; Bahaj et al., 2003).

$$
P=\frac{1}{2} \rho A V_{\text {marée }}^{3}
$$


Cependant, seule une partie de l'énergie disponible peut être captée par l'hydrolienne :

$$
P=\frac{1}{2} \rho C_{p} A V_{\text {marée }}^{3}
$$

Il est à noter que les expressions [1] et [2] sont valables pour tout système qui extrait l'énergie cinétique d'un fluide en mouvement (éoliennes et hydroliennes).

Pour les hydroliennes, le coefficient d'extraction d'énergie $C_{p}$ qui dépend à la fois de la vitesse du fluide et de la vitesse de rotation de la turbine est généralement défini dans l'intervalle 0,35-0,5 (Myers et al., 2006).

\subsubsection{Modèle de la ressource.}

La modélisation de la ressource nécessite la connaissance d'un certains nombre de données liées au site ciblé pour l'installation de l'hydrolienne (courant, bathymétrie, nature du fond). Les services hydrographiques tels que le SHOM en France disposent de bases de données où sont répertoriées, pour une zone donnée, les valeurs du courant en vives eaux et en mortes eaux. Ces valeurs sont indiquées pour chaque heure par rapport à la pleine mer, de H-6 à H+6. Connaissant le coefficient de marée, il est possible d'extrapoler la valeur du courant pour une heure donnée à partir du modèle du premier ordre suivant

$$
V_{\text {marée }}=V_{m e}+\frac{(C-45)\left(V_{v e}-V_{m e}\right)}{95-45}
$$

où 95 et 45 sont respectivement les coefficients de marée moyens correspondant aux vives eaux et mortes eaux. Cependant, les coefficients $\mathrm{C}, V_{m e}$ et $V_{v e}$ sont déterminés à partir des bases de données du SHOM (1994).

Afin de simplifier la modélisation, il était nécessaire d'adopter quelques hypothèses. En effet, l'écoulement est considéré, dans un premier temps, non perturbé. On néglige notamment les effets de la houle et de couches limites. On suppose aussi que la vitesse du courant est constante sur une heure. De plus, les tranches horaires, dans le livre des courants du SHOM, sont des heures marées. On rappelle qu'une heure marée est égale à 62 min. L'approximation reste donc correcte.

Pour illustrer cette méthodologie, nous avons calculé les vitesses des courants, dans le Raz-de-Sein, au cours de l'année 2007 et du mois de mars 2007. Les résultats obtenus sont présentés sur la figure 3 . 


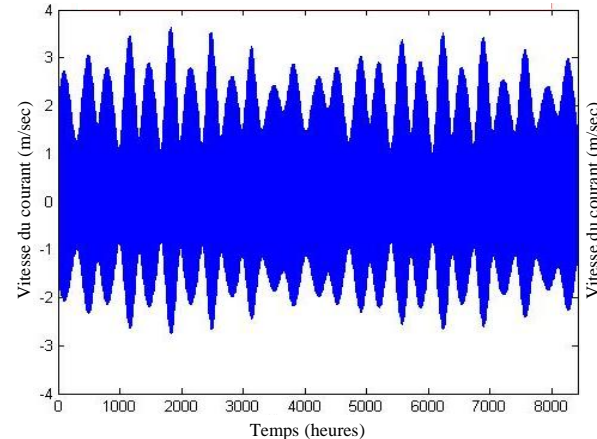

a) année 2007

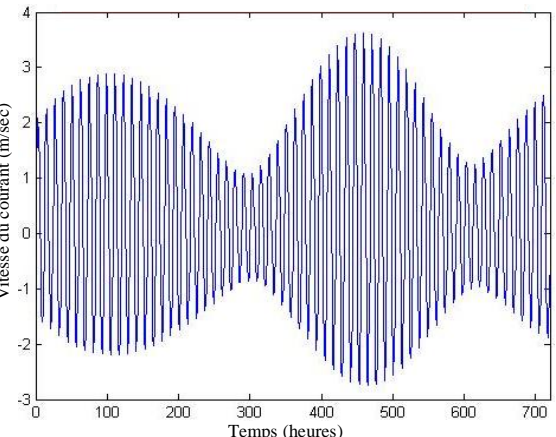

b) mars 2007

Figure 3. Vitesses des courants de marée dans le Raz de Sein pour l'année 2007 et le mois mars 2007

\subsection{Modélisation hydrodynamique de la turbine}

Afin d'être capable d'évaluer la quantité d'énergie extraite en fonction du régime de fonctionnement et de déterminer les lois de commande les plus favorables, il est nécessaire de disposer d'un modèle de performance du capteur d'énergie mécanique (turbine). En effet, ce modèle permet d'identifier la vitesse de rotation idéale de la turbine pour capter le maximum d'énergie. Notre travail sur ce sujet s'est focalisé sur le cas d'un capteur de type hélice; type le plus utilisé dans les technologies recensées (Ben Elghali et al., 2008 ; Bahaj et al., 2003).

La modélisation du capteur que nous avons intégrée est basée sur la théorie des quantités de mouvement (Blade Element Momentum - BEM) développée par Glauert. Cette théorie est une extension de la théorie de Rankine-Froude (Bossanyi et al., 2000 ; Batten et al., 2006). Elle repose sur le découpage selon le rayon de chaque pale en petits secteurs de dimension au rayon dr (figure 4) et sur le calcul itératif de leurs performances en termes de portance et traînée à partir des données hydrodynamiques d'un profil.

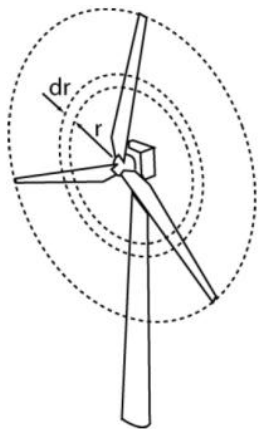

Figure 4. Découpage de la pale en secteurs élémentaires 
Il est alors possible en utilisant cette méthode de déterminer les coefficients d'extraction de puissance en fonction du régime et de la géométrie de l'hélice (profil, loi de vrillage, loi de corde, etc.). Cette méthodologie est décrite plus en détails chez (Ben Elghali et al., 2007). Ce modèle a été validé en comparant les résultats de simulations avec les données expérimentales existant dans la littérature (Ben Elghali et al., 2007 ; Bahaj et al., 2007).

Afin d'illustrer nos propos nous avons choisi de simuler une hélice à 3 pales utilisant un profil NACA44 (figure 5a) (Myers et al., 2005). Le profil des pales choisi doit en effet répondre à des contraintes de résistance mécanique avec des épaisseurs plus fortes que celles employées pour les éoliennes et des distributions de pression n'entraînant pas de phénomènes de cavitation préjudiciables. Ce profil offre un bon coefficient de portance pour différentes incidences ainsi qu'une faible trainée, ce qui autorise une large gamme de fonctionnement.

Le préprocesseur d'un code de calcul, développé par l'Institut de recherche de l'Ecole navale, dit Singularités, est utilisé pour visualiser l'allure du profil choisi (figure 5b). Ce code permet d'obtenir un maillage précis du capteur à partir de lois géométriques.

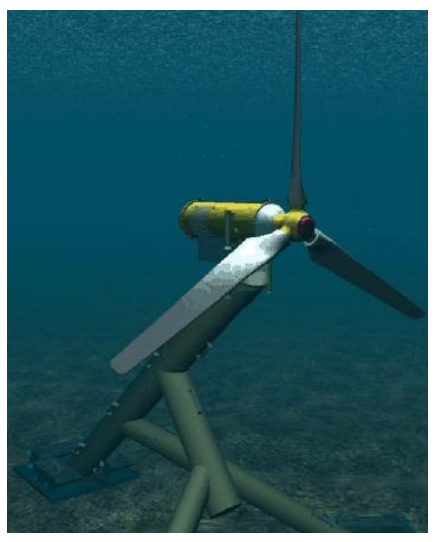

a)

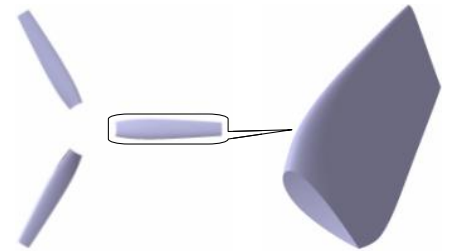

b)

Figure 5. a) exemple d'hydrolienne tripale [@Strøm AS] ; b) maillage des pales

L'algorithme ainsi défini permet de calculer les coefficients de puissance qui seront utilisés par la suite dans le bloc hydrodynamique de la turbine sous Matlab/Simulink®. Ces coefficients, illustrés par la figure 6a pour une hélice tripale de 1,44 $\mathrm{m}$ de diamètre, dépendent de la vitesse tangentielle $\lambda$ de la turbine et de la vitesse des courants marins $V_{\text {maree }}$ A partir de ces coefficients, une puissance utile peut être déduite figure $6 b$. 


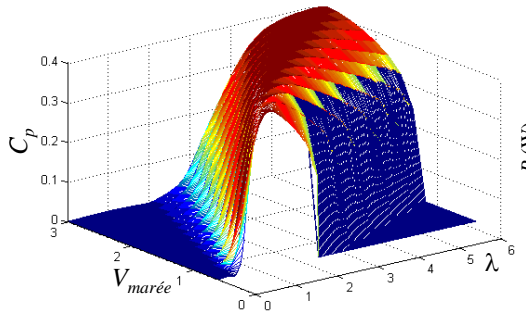

a)

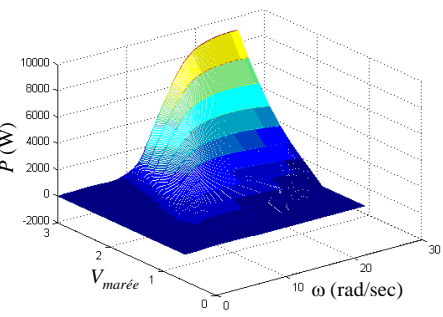

b)

Figure 6. a) coefficient de puissance $\left.C_{p}\left(\lambda, V_{\text {marée }}\right) ; b\right)$ puissance utile $P\left(\omega, V_{\text {marée }}\right)$ pour une hélice tripale de 1,44 $m$ de diamètre

\subsection{Modélisation de la génératrice}

La génératrice choisie pour la conversion de l'énergie hydrocinétique est la génératrice asynchrone à double alimentation (Müller et al., 2002 ; Park et al., 2004). De plus, on opte pour une GADA pilotée par le rotor avec une limitation de la plage de variation de vitesse de $\pm 50 \%$ de la vitesse nominale. Ce choix permet d'utiliser un seul convertisseur dimensionné pour une puissance nominale de l'ordre de 25 à $30 \%$ de la puissance nominale (Multon et al., 2003). Il sera donc moins volumineux, moins coûteux et nécessitera un système de refroidissement moins encombrant (Carrasco et al., 2006). Le convertisseur est alors modélisé par une source de tension contrôlée au niveau des enroulements de la machine. On considère en effet au premier ordre que le convertisseur MLI est capable de fournir des tensions aux bornes des enroulements rotoriques images des rapports cycliques en sortie de commande si l'on reste dans une gamme de fréquence et de tension compatible avec le bus continu du pont et la fréquence de commutation. Cette modélisation classique des convertisseurs qui néglige les phénomènes hautes fréquences liés à la MLI permet d'effectuer la simulation dans un temps raisonnable, le pas de calcul n'ayant pas à être adapté à la fréquence de commutation.

La modélisation de la GADA est décrite dans le référentiel de Park. Le système d'équation suivant [4] décrit la modélisation globale de la génératrice. Dans ce contexte, la figure 7 illustre le modèle de la génératrice utilisé pour calculer les correcteurs nécessaires à la commande en vitesse variable.

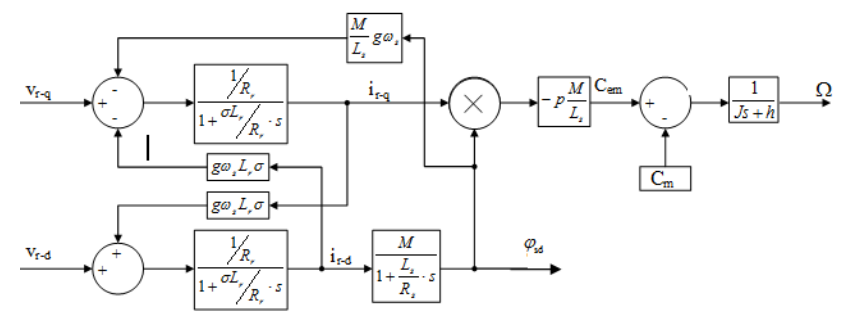


Figure 7. Modélisation de la génératrice asynchrone double alimentation

Nous pouvons établir les expressions des tensions statoriques et rotoriques dans le repère tournant $\mathrm{d}$-q quelconque :

$$
\begin{aligned}
& \mathrm{v}_{\mathrm{sd}}=\mathrm{R}_{\mathrm{s}} \cdot \mathrm{i}_{\mathrm{sd}}+\frac{\mathrm{d} \varphi_{\mathrm{sd}}}{\mathrm{dt}}-\frac{\mathrm{d} \theta_{\mathrm{s}}}{\mathrm{dt}} \varphi_{\mathrm{sq}} \\
& \mathrm{v}_{\mathrm{sq}}=\mathrm{R}_{\mathrm{s}} \cdot \mathrm{i}_{\mathrm{sq}}+\frac{\mathrm{d} \varphi_{\mathrm{sq}}}{\mathrm{dt}}+\frac{\mathrm{d} \theta_{\mathrm{s}}}{\mathrm{dt}} \varphi_{\mathrm{sd}} \\
& \mathrm{v}_{\mathrm{rd}}=\mathrm{R}_{\mathrm{r}} \cdot \mathrm{i}_{\mathrm{rd}}+\frac{\mathrm{d} \varphi_{\mathrm{rd}}}{\mathrm{dt}}-\frac{\mathrm{d} \theta_{\mathrm{r}}}{\mathrm{dt}} \varphi_{\mathrm{rq}} \\
& \mathrm{v}_{\mathrm{rq}}=\mathrm{R}_{\mathrm{r}} \cdot \mathrm{i}_{\mathrm{rq}}+\frac{\mathrm{d} \varphi_{\mathrm{rq}}}{\mathrm{dt}}-\frac{\mathrm{d} \theta_{\mathrm{r}}}{\mathrm{dt}} \varphi_{\mathrm{rd}}
\end{aligned}
$$

Les équations électriques liant les différents courants $\left(i_{s i}, i_{r i}, i \in(d, q)\right)$ aux flux $\left(\varphi_{s i}, \varphi_{r i}, i \in(d, q)\right)$ statoriques et rotoriques qui interagissent dans la machine sont définies par :

$$
\begin{aligned}
& \varphi_{\mathrm{sd}}=\mathrm{L}_{\mathrm{s}} \cdot \mathrm{i}_{\mathrm{sd}}+\mathrm{M}_{\mathrm{sr}} \cdot \mathrm{i}_{\mathrm{rd}} \\
& \varphi_{\mathrm{sq}}=\mathrm{L}_{\mathrm{s}} \cdot \mathrm{i}_{\mathrm{sq}}+\mathrm{M}_{\mathrm{sr}} \cdot \mathrm{i}_{\mathrm{rq}} \\
& \varphi_{\mathrm{rd}}=\mathrm{L}_{\mathrm{r}} \cdot \mathrm{i}_{\mathrm{rd}}+\mathrm{M}_{\mathrm{sr}} \cdot \mathrm{i}_{\mathrm{sd}} \\
& \varphi_{\mathrm{rq}}=\mathrm{L}_{\mathrm{r}} \cdot \mathrm{i}_{\mathrm{rq}}+\mathrm{M}_{\mathrm{sr}} \cdot \mathrm{i}_{\mathrm{sq}}
\end{aligned}
$$

Le couple électromagnétique de la machine peut s'exprimer sous différentes formes. Celle qui sera utilisée pour concevoir le système de commande est définie par:

$$
\begin{aligned}
& \mathrm{C}_{\mathrm{em}}=\mathrm{p} \cdot \mathrm{M}\left(\mathrm{I}_{\mathrm{qs}} \cdot \mathrm{I}_{\mathrm{dr}}-\mathrm{I}_{\mathrm{ds}} \cdot \mathrm{i}_{\mathrm{qr}}\right) \\
& \mathrm{C}_{\mathrm{em}}=\mathrm{p} \cdot\left(\varphi_{\mathrm{sd}} \cdot \mathrm{i}_{\mathrm{sq}}-\varphi_{\mathrm{sq}} \cdot \mathrm{i}_{\mathrm{sd}}\right)
\end{aligned}
$$

L'équation mécanique du système est définie par :

$$
J \frac{d \omega}{d t}=C_{e m}-C_{m}-f \omega
$$

Le couple mécanique $\mathrm{C}_{\mathrm{m}}$ est la sortie du bloc de modélisation de la turbine tel que décrit à la figure 2 . 
Les paramètres de la génératrice asynchrone double alimentation utilisés dans la simulation sont :

$R_{s}=0.455 \Omega, L_{s}=0.084 \mathrm{H}, R_{r}=0.62 \Omega, L_{r}=0.081 \mathrm{H}, M=0.078 \mathrm{H}, J=0.3125$ $\mathrm{kg} . \mathrm{m}^{2}, f=6.7310^{-3} \mathrm{Nms}^{-1}$.

\section{Stratégie de commande de la génératrice asynchrone double alimentation}

Pour des raisons évidentes de simplifications, un référentiel $d-q$ lié au champ tournant statorique et un flux statorique aligné sur l'axe $d$ ont été adoptés $\varphi_{s q}=0$ (Müller et al., 2002). De plus, la résistance statorique peut être négligée étant donné que c'est une hypothèse réaliste pour les génératrices utilisées dans l'éolien (Tapia et al., 2003 ; 2007). Partant de ces considérations, le couple devient :

$$
\mathrm{C}_{\mathrm{em}}=\mathrm{p} \cdot \varphi_{\mathrm{sd}} \cdot \mathrm{i}_{\mathrm{sq}}
$$

Pour la synthèse de la commande on a choisi un fonctionnement à perte cuivre minimal. Les pertes cuivre totales $\left(P_{c u}\right)$ dans la GADA ont pour expression :

$$
\mathrm{P}_{\mathrm{cu}}=\mathrm{R}_{\mathrm{s}}\left(\mathrm{i}_{\mathrm{sd}}{ }^{2}+\mathrm{i}_{\mathrm{sq}}{ }^{2}\right)+\mathrm{R}_{\mathrm{r}}\left(\mathrm{i}_{\mathrm{rd}}{ }^{2}+\mathrm{i}_{\mathrm{rq}}{ }^{2}\right)
$$

En substituant les courants $i_{r d}$ et $i_{r q}$ par leur expression, et en dérivant $P_{c u}$ par rapport à $i_{s d}$, on obtient le minimum de pertes pour :

$$
\begin{gathered}
\mathrm{i}_{\mathrm{sd}}=\frac{\mathrm{L}_{\mathrm{s}} \mathrm{R}_{\mathrm{r}}}{\mathrm{R}_{\mathrm{s}} \mathrm{M}^{2}+\mathrm{R}_{\mathrm{r}} \mathrm{L}_{\mathrm{s}}^{2}} \varphi_{\mathrm{sd}} \\
\text { soit } \quad \mathrm{i}_{\mathrm{rd} \_ \text {ref }}=\frac{\mathrm{MR}_{\mathrm{s}}}{\mathrm{R}_{\mathrm{s}} \mathrm{M}^{2}+\mathrm{R}_{\mathrm{r}} \mathrm{L}_{\mathrm{s}}^{2}} \varphi_{\text {sd_ref }}
\end{gathered}
$$

La commande de la GADA doit permettre la génération du maximum de puissance en fonction de la vitesse des courants de marée au moyen d'une stratégie MPPT (figure 7) (Koutroulis et al., 2009). La consigne de puissance réactive sera maintenue nulle de façon à garder un facteur de puissance unitaire côté stator.

Dans un repère diphasé quelconque, la puissance réactive statorique d'une génératrice asynchrone s'écrit :

$$
\mathrm{Q}_{\mathrm{s}}=\mathrm{V}_{\mathrm{qs}} \mathrm{I}_{\mathrm{ds}}-\mathrm{V}_{\mathrm{ds}} \mathrm{I}_{\mathrm{qs}}
$$

L'adaptation de cette équation aux hypothèses simplificatrices donne : 


$$
\mathrm{Q}_{\mathrm{s}}=\frac{\mathrm{V}_{\mathrm{s}}}{\mathrm{L}_{\mathrm{s}}}\left(\varphi_{\mathrm{sd}}-\mathrm{MI}_{\mathrm{dr}}\right)
$$

soit avec les hypothèses de l'étude $Q_{s}=0$ :

$$
\mathrm{i}_{\text {rd_ref }}=\frac{\varphi_{\text {sd_ref }}}{\mathrm{M}}
$$

La stratégie de commande est schématiquement illustrée par la figure 8.

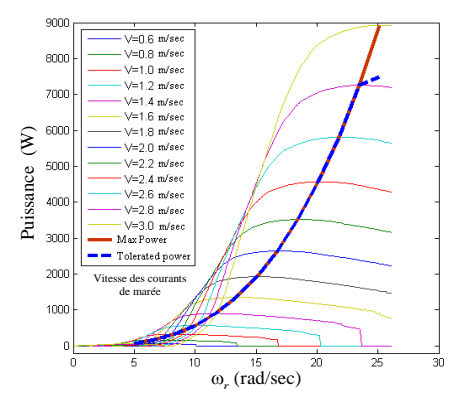

Figure 8. Courbes de puissances en fonction des vitesses de courants de marée et loi de vitesse de rotation optimale (MPPT)

Sur la plage de fonctionnement de la GADA (vitesse nominale $\pm 50 \%$ ). La vitesse de la génératrice est contrôlée afin de suivre la loi décrite par la figure 8 (MPPT). Dans ce cas, il y a adaptation de la vitesse de la génératrice à celle du fluide afin d'extraire le maximum de puissance pour chaque régime de courant de marée. Ainsi, à chaque vitesse du fluide correspond une vitesse de rotation «optimale » de la turbine qui permet d'extraire un maximum d'énergie (lieu des maxima des $\mathrm{Cp}$ ) Cette vitesse peut être facilement calculée pour chaque valeur de courant après établissement du modèle hydrodynamique de la turbine. Il faut noter que la mise en œuvre de ce type de commande nécessite soit l'acquisition de la vitesse du fluide par un capteur soit son estimation en utilisant par exemple le modèle décrit dans la sous-section 2.1).

Le bloc MPPT qui permet de déterminer une vitesse optimale de référence à partir de la vitesse du fluide (figure 9) permet de générer des vitesses de références à partir des courants de marée. En effet, en se plaçant sur les maximaux des courbes (figure 7), on peut déterminer la vitesse de rotation de la turbine qui peut assurer l'extraction du maximum de puissance pour une vitesse de marée donnée. Dans le but d'assurer la régulation, des correcteurs PI sont utilisés. La figure 10 décrit la structure de la commande en vitesse adaptée.

Avec $k$ une constante définie par l'expression $\mathrm{K}=-\frac{\mathrm{L}_{\mathrm{s}}}{\mathrm{p} \cdot \mathrm{M} \cdot \Phi_{\mathrm{sd}}}$. 
Les gains des régulateurs PI utilisés sont choisis élevés afin que le système global de régulation des courants soit suffisamment rapide en vue des régulations de vitesse qui en dépendent directement.

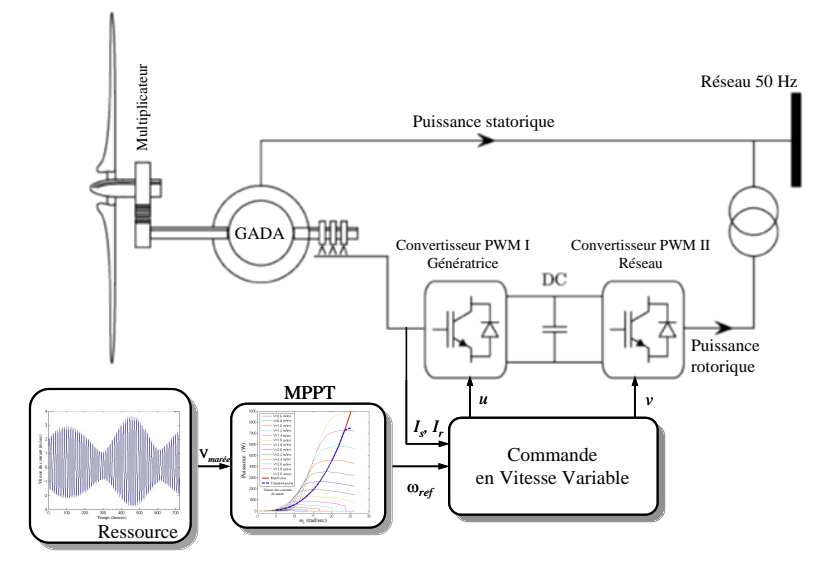

Figure 9. Principe de la stratégie de commande

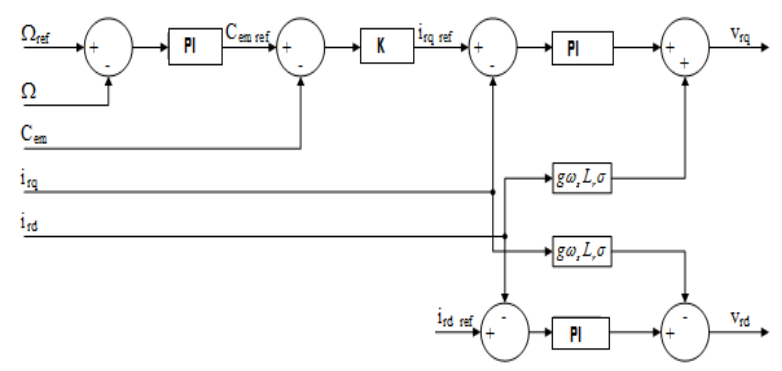

Figure 10. Structure de la commande en vitesse

\section{Exemple d'illustration et de validation}

\subsection{Description de l'hydrolienne}

Le choix du site d'implantation de l'hydrolienne doit satisfaire à plusieurs critères : vitesse des courants de marée (Myers et al., 2006 ; EU Commission, 1996), bathymétrie du site, nature du sol et distance par rapport à la terre (Batten et al., 2006). Notre choix, pour illustrer l'utilisation de l'outil, s'est ainsi porté sur le site du Raz-de-Sein (figure 11) parmi plusieurs sites cités dans le rapport EUR16683 Commission européenne (EU Commission, 1996). 


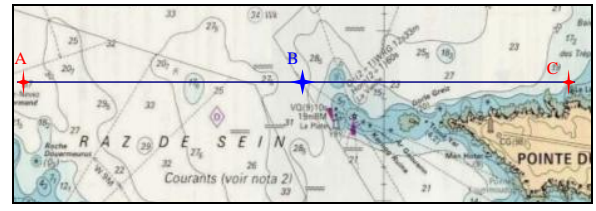

Figure 11. Site choisi pour l'installation de l'hydrolienne

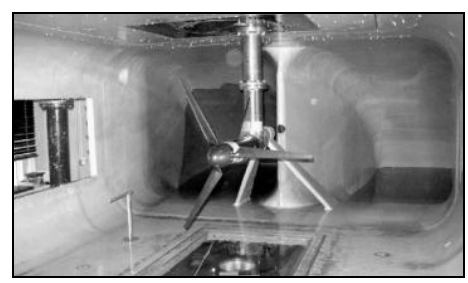

Figure 12. L'hydrolienne testée (Ben Elghali et al., 2007 ; Bahaj et al., 2007)

L'hydrolienne utilisée pour valider l'outil de simulation est équipée d'un capteur de $1,44 \mathrm{~m}$ de diamètre (figure 12) et d'une génératrice asynchrone à 4 pôles et à double alimentation de $7,5 \mathrm{~kW}$ dont les paramètres sont fournis en sous-section 2.3. Cette machine est connectée à un réseau $50 \mathrm{~Hz}$. Il faut noter que le capteur choisi correspond à celui de la figure $4 \mathrm{~b}$.

\subsection{Ressource non perturbée}

Dans un premier temps, les simulations sont réalisées pour un modèle du premier ordre de la ressource [3]. Cette dernière est supposée non affectée par les perturbations du milieu marin (vagues, houle, etc.).

Les résultats de simulations sont illustrés par les figures 13 et 14 (vitesse et puissance générées). Dans ce premier, la vitesse de référence pour la stratégie MPPT est identique à celle du fluide. Il faut noter que les essais ont été effectués sur une échelle de temps réduite pour des raisons de temps de calcul où un cycle de marée est ramené à une période de l'ordre de la dizaine de secondes. D'autre part, dans ces premières simulations, la limitation en vitesse $( \pm 50 \%$ de la vitesse de synchronisme $)$ est prise en compte.

Les résultats obtenus montrent un bon suivi des vitesses de référence. Cependant, il subsiste une erreur de $10 \%$ sur la puissance active simulée. Ces résultats montrent qu'il serait sans doute intéressant en termes de perspective d'utiliser une commande directe de la puissance active (Lie et al., 2006). 


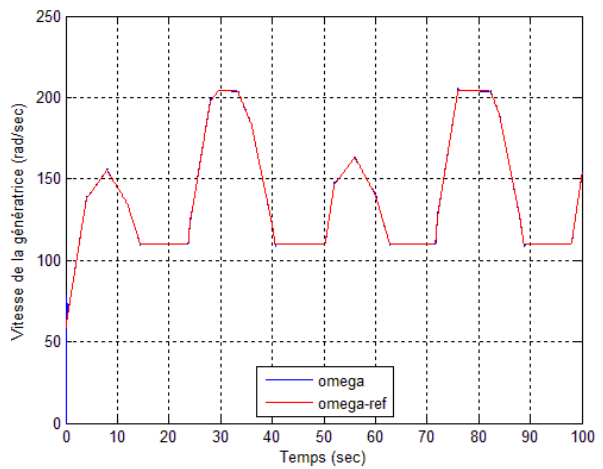

Figure 13. Vitesse de la génératrice

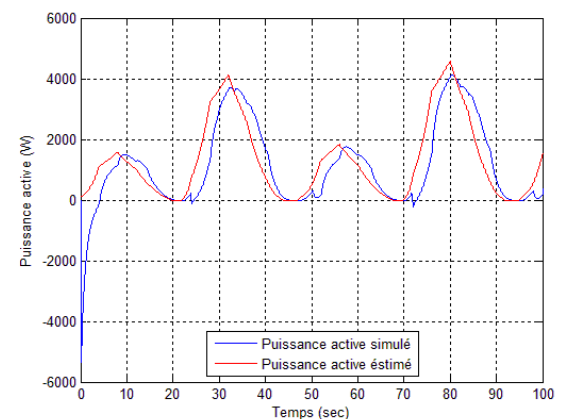

Figure 14. Puissance active de la génératrice

\subsection{Ressource perturbée}

Afin d'évaluer le modèle de la ressource et son effet sur la puissance générée, trois types de ressources ont été utilisés en entrée du modèle pour prendre en compte des perturbations externes telles qu'illustré par le tableau 1. Dans le cas étudié, nous considérons une période de 90 secondes ou la vitesse moyenne prédite par l'équation [3] peut être considérée comme constante et égale à $2 \mathrm{~m} / \mathrm{sec}$ (4 nœuds). Ainsi les trois types de ressources considérées sont composées par: la vitesse du courant de marée, la vitesse générée par la houle, et un signal aléatoire $b(t)$ qui représente toutes les autres perturbations. La vitesse de la génératrice est dans ces simulations limitée à $\pm 50 \%$ de la vitesse de synchronisme (entre 78 et $235 \mathrm{rad} / \mathrm{sec}$ ).

Pour prendre en compte l'effet de la houle qui est a priori considérée comme l'élément le plus perturbateur pour la ressource, le modèle de Stokes est adopté. Il 
permet de calculer, pour une houle théorique donnée de période $T$, d'amplitude $H$ et de longueur d'onde $L$ se propageant sur un fond de profondeur $d$ (figure 15), la vitesse induite à une profondeur donnée $z$ selon l'expression très connue [9].

$$
\left\{\begin{array}{l}
V_{\text {tides }}=\operatorname{grad} \phi \\
\phi=-\frac{H L}{2 T} \frac{\operatorname{ch} 2 \Pi\left(\frac{z+d}{L}\right)}{\operatorname{sh} 2 \Pi\left(\frac{d}{L}\right)} \sin 2 \Pi\left(\frac{t}{T}-\frac{x}{L}\right)
\end{array}\right.
$$

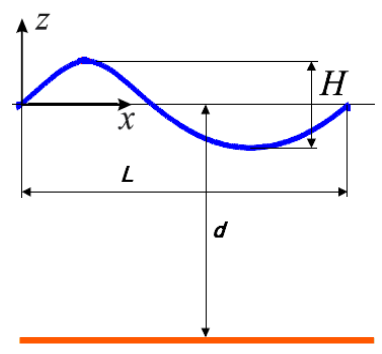

Figure 15. Caractéristiques de la houle

\begin{tabular}{|c|c|c|}
\hline $\begin{array}{c}\text { Case } \\
\mathrm{s}\end{array}$ & $\begin{array}{l}\text { Conditions de simulations/vitesse du courant } \\
\qquad(\mathrm{m} / \mathrm{sec})\end{array}$ & $\begin{array}{c}\mathrm{P} \\
(\mathrm{kW})\end{array}$ \\
\hline 1 & $\begin{array}{c}\left.V_{\text {real }}=2 \text { (pas de perturbation }\right) \\
V_{\text {ref }}=2\end{array}$ & 2.535 \\
\hline 2 & $\begin{array}{l}V_{\text {real }}=2+0.3252 \cos (0.4189 t)+0.2749 \cos (0.6283 t) \\
V_{\text {ref }}=2+0.3252 \cos (0.4189 t)+0.2749 \cos (0.6283 t)\end{array}$ & 2.694 \\
\hline 3 & $\begin{array}{c}V_{\text {real }}=2+0.3252 \cos (0.4189 t)+0.2749 \cos (0.6283 t) \\
V_{\text {ref }}=2\end{array}$ & 2.612 \\
\hline 4 & $\begin{array}{c}V_{\text {real }}=2+0.3252 \cos (0.4189 t)+0.2749 \cos (0.6283 t) \\
+b(t) \quad V_{\text {ref }}=2\end{array}$ & 2.691 \\
\hline 5 & $\begin{aligned} V_{\text {real }}= & 2+0.3252 \cos (0.4189 t)+0.2749 \cos (0.6283 t) \\
& +b(t) \\
V_{\text {real }}= & 2+0.3252 \cos (0.4189 t)+0.2749 \cos (0.6283 t)\end{aligned}$ & 2.821 \\
\hline
\end{tabular}

Tableau 1. Cas simulés 
Cette vitesse est alors additionnée à la vitesse du courant, ce qui revient à négliger les interactions croisées entre la houle et le courant. Dans le futur, nous comptons également utiliser dans nos simulations des mesures réelles ou issues de calculs numériques qui prennent en compte cette interaction, ce qui nous permettrait d'accéder à un meilleur niveau de réalisme.

Différents types de vitesse de courant servent de référence pour déterminer la consigne en vitesse de rotation en fonction de la stratégie MPPT selon que l'on considère que cette vitesse de référence est issue d'une mesure par un capteur (cas 1, 2 et 5) ou d'une estimation par le modèle de premier ordre décrit en soussection 2.1 (cas 3 et 4$)$.
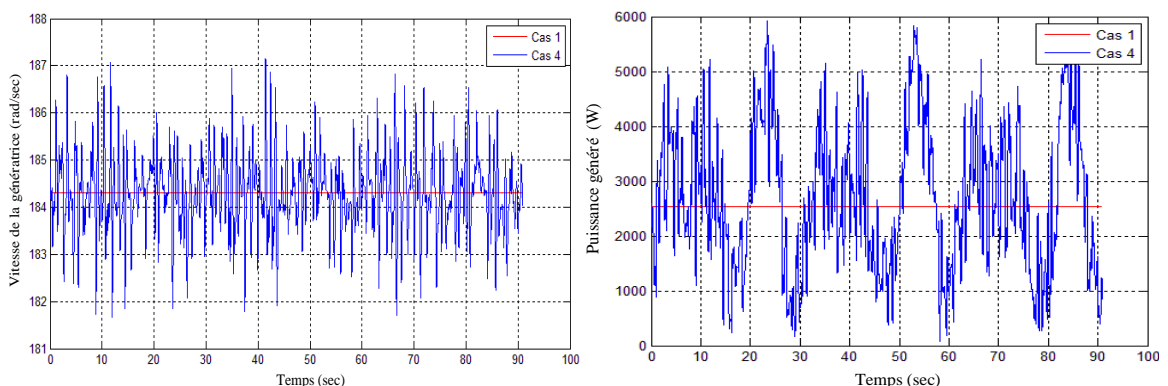

a) cas 1 et 4
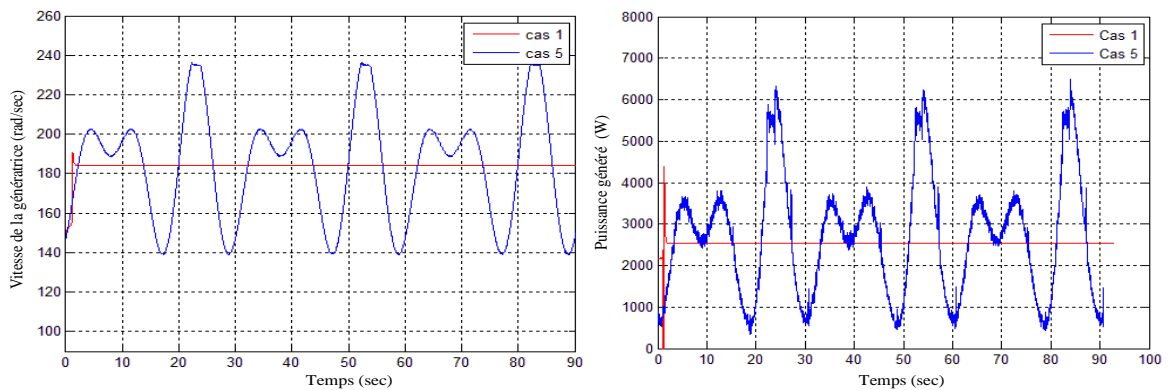

b) cas 1 et 5
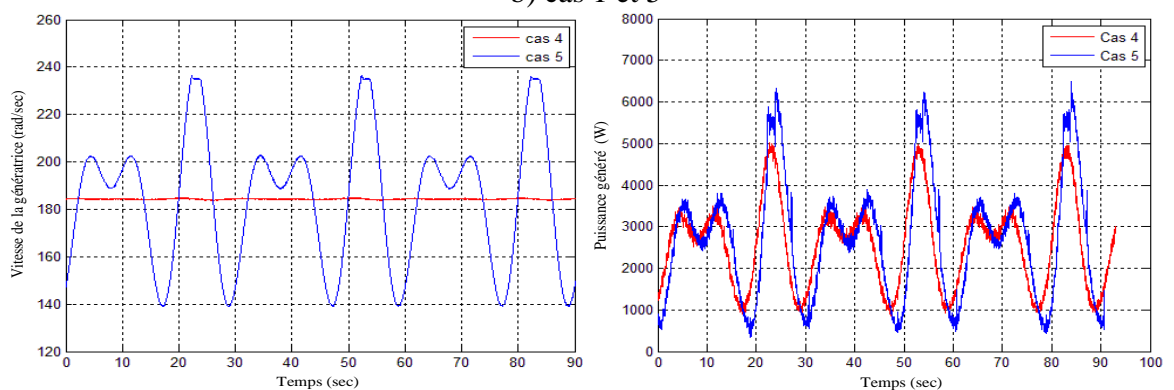

c) cas 4 et 5

Figure 16. Performances de la génératrice pour une ressource perturbée 
La figure 16 illustre alors la vitesse de la génératrice ainsi que la puissance qu'elle génère les différents cas du tableau 1. Les résultats obtenus par simulation montrent que le modèle du premier ordre proposé pour la ressource est suffisamment efficace comme élément d'entrée du bloc MPPT. Il peut en outre permettre l'extraction de $95 \%$ de la puissance utile potentielle (cas 5); et ceci même si la ressource est perturbée. De plus, avec ce type de modélisation, on peut constater que la génératrice asynchrone est moins sollicitée en termes de fluctuation de puissance.

\section{Conclusions}

Dans cet article, une modélisation et une commande d'un système de récupération de l'énergie des courants de marée (hydrolienne) équipée d'une génératrice asynchrone à double alimentation ont été proposées. Dans un premier temps, un modèle multiphysique de l'hydrolienne est élaboré sous Matlab/Simulink®. Ce modèle comprend un modèle de la ressource, de la turbine, de la génératrice et de sa commande. Le modèle hydrodynamique de la turbine est validé en comparant les résultats de simulations avec les données expérimentales existant dans la littérature. Ensuite, une stratégie de commande de la génératrice asynchrone est proposée, permettant d'extraire le maximum de puissance en fonction de la vitesse du courant. Le comportement de l'hydrolienne ainsi modélisé est simulé en commande en vitesse variable pour une puissance de génératrice de 7,5 kW en utilisant des données réelles du site du Raz-de-Sein pour l'année 2007. Les résultats obtenus sont assez satisfaisants en termes de suivi de vitesse mais suggèrent l'utilisation future d'une commande directe de la puissance active. Pour ce qui est de l'effet des perturbations du milieu marin, les résultats obtenus montrent que le modèle du premier ordre proposé pour la ressource est suffisamment efficace comme élément d'entrée du bloc MPPT. Il est à noter qu'une validation expérimentale de ce modèle est envisagée dans les prochains mois sur un banc expérimental permettant l'émulation de la ressource et de la turbine.

\section{Remerciement}

Ce travail est soutenu par Brest métropole océan (BMO) et le Fond social européen (FSE). Il est aussi soutenu par le GDR SEEDS CNRS N²994, dans le cadre du projet interne HYDROLE.

\section{Bibliographie}

2005 IEEE Power Engineering Society General Meeting Panel Session, "Harnessing the untapped energy potential of the oceans: Tidal, wave, currents and OTEC," San Francisco (USA), June 2005. 
Johnstone C.M., et al., "EC FPVI co-ordinated action on ocean energy: A European platform ford sharing technical information and research outcomes in wave and tidal energy systems", Renewable Energy, vol. 31, 2006, p. 191-196.

Ben Elghali S.E., et al., «Les systèmes de génération d'énergie électriques à partir des courants de marée », Revue $3 E I, \mathrm{n}^{\circ}$ 52, mars 2008, p. 73-85.

Benbouzid M.E.H., et al., "Marine tidal current electric power generation technology: State of the art and current status," Proceedings of IEEE IEMDC'07, Antalya (Turkey), vol. 2, May 2007, p. 1407-1412.

Myers L., et al., "Simulated electrical power potential harnessed by marine current turbine arrays in the Alderney Race", Renewable Energy, vol. 30, 2005, p. 1713-1731.

Couch J.S., et al., "Tidal current energy extraction: Hydrodynamic resource characteristics", Proc. IMechE, Part M: Journal of Engineering for the Maritime, vol. 220, n 4, 2006, p. 185-194.

Bahaj A.S., et al., "Fundamentals applicable to the utilisation of marine current turbines for energy production", Renewable Energy, vol. 28, 2003, p. 2205-2211.

Myers L., et al., "Power output performance characteristics of a horizontal axis marine current turbine", Renewable Energy, vol. 31, 2006, p. 197-208.

Bossanyi E., Wind Energy Handbook, New York, Wiley, 2000.

Batten W.M.J., et al., "Hydrodynamics of marine current turbines", Renewable Energy, vol. 31, 2006, p. 249-256.

Ben Elghali et al., "A simulation model for the evaluation of the electrical power potential harnessed by a marine current turbine", IEEE Journal on Oceanic Engineering, vol. 32, $\mathrm{n}^{\circ}$ 4, October 2007, p. 786-797.

Bahaj A.S., et al., "Power and thrust measurements of marine current turbines under various hydrodynamic flow conditions in a cavitation tunnel and a towing tank", Renewable Energy, vol. 32, 2007, p. 407-426.

Müller S., et al., "Doubly fed induction generator systems", IEEE Industry Applications Magazine, vol. 8, n 3, May-June 2002, p. 26-33.

Park J.W., et al., "Wide speed operation of a doubly-fed induction generator for tidal current energy", Proceedings of the IEEE IECON'2004, Busan, South Korea, vol. 2, 2004, p. 1333-1338.

Multon B., et al., "Le génie électrique dans le vent : Etat de l'art dans le domaine de la génération éolienne”, JCGE'03, Saint-Nazaire, juin 2003.

Carrasco J.M., et al., "Power-electronic systems for the grid integration of renewable energy sources: A survey", IEEE Trans. Industrial Electronics, vol. 53, n 4, June 2006, p. $1002-$ 1016.

Tapia G., et al., "Proportional-integral regulator-based approach to wind farm reactive power management for secondary voltage control", IEEE Trans. Energy Conversion, vol. 22, $\mathrm{n}^{\circ} 2$, June 2007, p. 488-498. 
Tapia A., et al., "Modeling and control of a wind turbine driven doubly fed induction generator", IEEE Trans. Energy Conversion, vol. 18, n 2, June 2003, p. 194-204.

Koutroulis E., et al., "Design of a maximum power tracking system for wind-energyconversion applications", IEEE Trans. Industrial Electronics, vol. 53, n² 2, April 2006, p. 486-494.

EU Commission, The exploitation of tidal marine currents, Report EUR16683EN, 1996.

Lie X., et al., "Direct active and reactive power control of DFIG for wind energy generation", IEEE Trans. Energy Conversion, vol. 21, n 3, September 2006, p. 750-758.

SHOM. Courants de marée de la côte ouest de Bretagne : de Goulven à Penmarc'h, Ed : SHOM Paris, novembre 1994.

\section{Nomenclature}

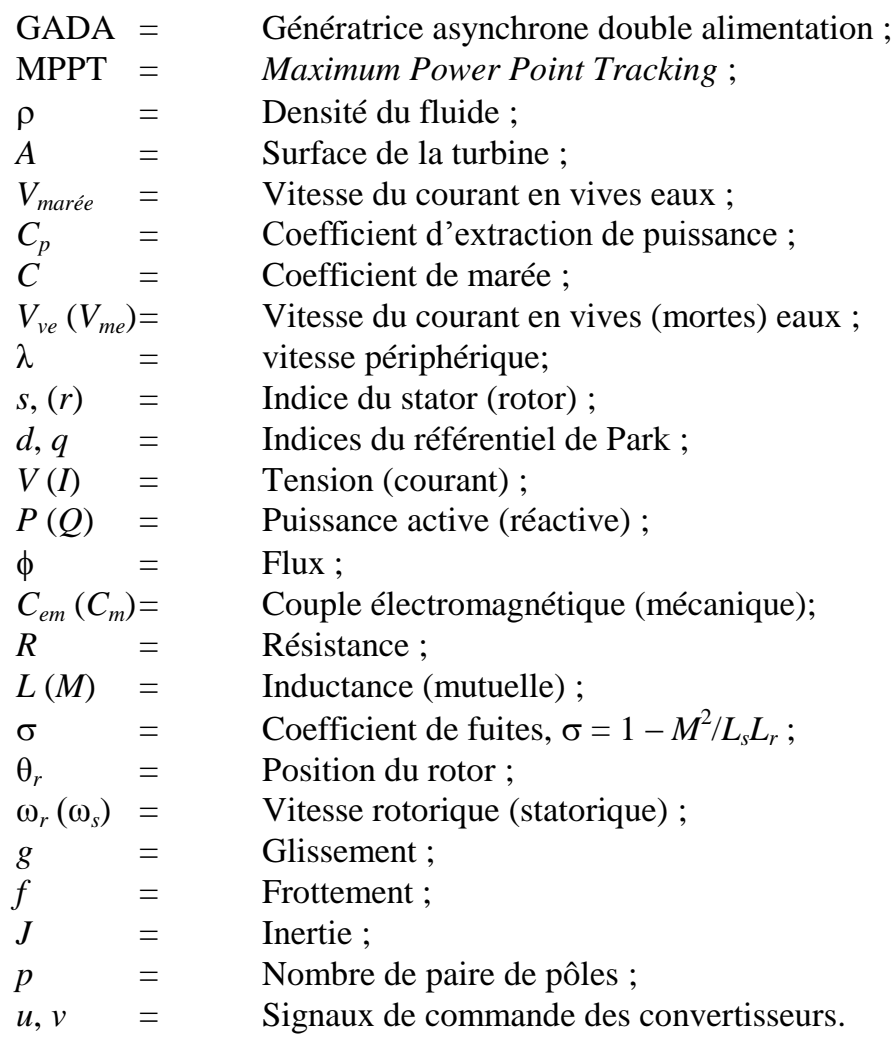

